Research Article

\title{
Boundedness of Marcinkiewicz Integrals and Their Commutators on Generalized Weighted Morrey Spaces
}

\author{
Runqing Cui ${ }^{1}$ and $\mathrm{Zhang} \mathrm{Li}^{2}$ \\ ${ }^{1}$ College of Mathematics and Informatics, Henan Polytechnic University, Jiaozuo 454003, China \\ ${ }^{2}$ College of Computer Science and Technology, Henan Polytechnic University, Jiaozuo 454003, China \\ Correspondence should be addressed to Runqing Cui; lgdcuirunqing@yeah.net \\ Received 22 September 2014; Accepted 12 December 2014 \\ Academic Editor: Lars E. Persson
}

Copyright (c) 2015 R. Cui and Z. Li. This is an open access article distributed under the Creative Commons Attribution License, which permits unrestricted use, distribution, and reproduction in any medium, provided the original work is properly cited.

We will study the boundedness of Marcinkiewicz integrals with rough kernel on the generalized weighted Morrey spaces. We will also prove that the commutator operators formed by a $B M O\left(\mathbb{R}^{n}\right)$ function and Marcinkiewicz integrals are also bounded on the generalized weighted Morrey spaces.

\section{Introduction and Results}

Suppose that $\mathbb{S}^{n-1}$ is the unit sphere in $\mathbb{R}^{n}(n \geq 2)$ equipped with the normalized Lebesgue measure $d \sigma$. Let $\Omega \in L^{q}\left(\mathbb{S}^{n-1}\right)$ with $1<q \leq \infty$ be homogeneous of degree zero and satisfy the cancellation condition

$$
\int_{\mathbb{S}^{n-1}} \Omega\left(x^{\prime}\right) d \sigma\left(x^{\prime}\right)=0
$$

where $x^{\prime}=x /|x|$ for any $x \neq 0$. The Marcinkiewicz integral of higher dimension $\mu_{\Omega}$ is defined by

$$
\mu_{\Omega}(f)(x)=\left(\int_{0}^{\infty}\left|F_{\Omega, t}(x)\right|^{2} \frac{d t}{t^{3}}\right)^{1 / 2}
$$

where

$$
F_{\Omega, t}(x)=\int_{|x-y| \leq t} \frac{\Omega(x-y)}{|x-y|^{n-1}} f(y) d y
$$

We will also consider the commutator generated by Marcinkiewicz integral $\mu_{\Omega}$ and $b$ is defined as follows:

$$
\begin{aligned}
& {\left[b, \mu_{\Omega}\right](f)(x)} \\
& =\left(\int_{0}^{\infty} \mid \int_{|x-y| \leq t} \frac{\Omega(x-y)}{|x-y|^{n-1}}\right. \\
& \left.\left.\quad \cdot(b(x)-b(y)) f(y) d y\right|^{2} \frac{d t}{t^{3}}\right)^{1 / 2} .
\end{aligned}
$$

The following results concerning the boundedness of Marcinkiewicz integrals and their commutators on weighted $L^{p}$ space are known.

Theorem 1 (see [1]). Suppose that $\Omega \in L^{q}\left(\mathbb{S}^{n-1}\right)$ with $1<q \leq$ $\infty$. Then, for every $q^{\prime}<p<\infty$ and $\omega \in A_{p / q^{\prime}}$, there is a constant $C$ independent of $f$ such that

$$
\left\|\mu_{\Omega}(f)\right\|_{L^{p}\left(\omega, \mathbb{R}^{n}\right)} \leq C\|f\|_{L^{p}\left(\omega, \mathbb{R}^{n}\right)} .
$$

Theorem 2 (see [2]). Suppose that $\Omega \in L^{q}\left(\mathbb{S}^{n-1}\right)$ with $1<$ $q \leq \infty$ and $b \in B M O\left(\mathbb{R}^{n}\right)$. Then, for every $q^{\prime}<p<\infty$ and $\omega \in A_{p / q^{\prime}}$, there is a constant $C$ independent of $f$ such that

$$
\left\|\left[b, \mu_{\Omega}\right](f)\right\|_{L^{p}\left(\omega, \mathbb{R}^{n}\right)} \leq C\|b\|_{*}\|f\|_{L^{p}\left(\omega, \mathbb{R}^{n}\right)} .
$$


The classical Morrey spaces, introduced by Morrey [3] in 1938, have been studied intensively by various authors and together with weighted Lebesgue spaces play an important role in the theory of partial differential equations; they appeared to be quite useful in the study of local behavior of the solutions of elliptic differential equations and describe local regularity more precisely than Lebesgue spaces. See [47] for details.

Given $f \in L_{\text {loc }}^{p}\left(\mathbb{R}^{n}\right), 1 \leq p<\infty$ and $0 \leq \lambda \leq n$. Morrey spaces are defined by

$$
\|f\|_{L^{p, \lambda}\left(\mathbb{R}^{n}\right)}=\sup _{x \in \mathbb{R}^{n}, r>0} r^{-\lambda / p}\|f\|_{L^{p}(B(x, r))}<\infty .
$$

Note that $L^{p, 0}\left(\mathbb{R}^{n}\right)=L^{p}\left(\mathbb{R}^{n}\right)$ and $L^{p, n}\left(\mathbb{R}^{n}\right)=L^{\infty}\left(\mathbb{R}^{n}\right)$. If $\lambda<0$ or $\lambda>n$, then $L^{p, \lambda}\left(\mathbb{R}^{n}\right)=\Theta$, where $\Theta$ is the set of all functions equivalent to 0 on $\mathbb{R}^{n}$.

In [8] Chiarenza and Frasca show the boundedness of the Hardy-Littlewood maximal operator in $L^{p, \lambda}\left(\mathbb{R}^{n}\right)$ that allows them to prove continuity of fractional and classical CalderónZygmund operators in these spaces. Recall that integral operators of that kind appear in the representation formulae of the solutions of elliptic/parabolic equations and systems. Thus the continuity of the Calderón-Zygmund integrals implies regularity of the solutions in the corresponding spaces.

Let $\Phi(r), r>0$, be a growth function, that is, a positive increasing function in $(0, \infty)$, and satisfy doubling condition

$$
\Phi(2 r) \leq D \Phi(r), \quad \forall r>0,
$$

where $D=D(\Phi) \geq 1$ is a doubling constant independent of $r$. In [9] Mizuhara gave generalization Morrey spaces $L^{p, \Phi}\left(\mathbb{R}^{n}\right)$ considering $\Phi(r)$ instead of $r^{\lambda}$ in (7). He studied also a continuity in $L^{p, \Phi}\left(\mathbb{R}^{n}\right)$ of some classical integral operators.

Komori and Shirai [10] introduced a version of the weighted Morrey space $L^{p, \kappa}\left(\omega, \mathbb{R}^{n}\right)$, which is a natural generalization of the weighted Lebesgue space $L^{p}\left(\omega, \mathbb{R}^{n}\right)$. Let $1 \leq$ $p<\infty, 0<\kappa<1$ and let $\omega$ be a weight function. Then the spaces $L^{p, \kappa}\left(\omega, \mathbb{R}^{n}\right)$ are defined by

$$
\begin{aligned}
& \|f\|_{L^{p, \kappa}\left(\omega, \mathbb{R}^{n}\right)} \\
& \quad=\sup _{x \in \mathbb{R}^{n}, r>0}\left(\frac{1}{\omega(B(x, r))^{\kappa}} \int_{B(x, r)}|f(y)|^{p} \omega(y) d y\right)^{1 / p} \\
& \quad<\infty .
\end{aligned}
$$

Recently, Aliev and Guliev in [11] introduced another generalization of the Morrey spaces. Let $1 \leq p<\infty$ and let $\varphi$ be a positive measurable function on $\mathbb{R}^{n} \times(0, \infty)$. We denote by $M_{\varphi}^{p}\left(\mathbb{R}^{n}\right)$ the generalized Morrey space, the space of all functions $f \in L_{\mathrm{loc}}^{p}\left(\mathbb{R}^{n}\right)$ with finite norm

$$
\|f\|_{M_{\varphi}^{p}\left(\mathbb{R}^{n}\right)}=\sup _{x \in \mathbb{R}^{n}, r>0} \frac{1}{\varphi(x, r)}\left(\frac{1}{|B(x, r)|}\|f\|_{L^{p}(B(x, r))}^{p}\right)^{1 / p} .
$$

They also studied in [11] the boundedness of $\mu_{\Omega}$ and their commutator on generalized Morrey space $M_{\varphi}^{p}\left(\mathbb{R}^{n}\right)$ when $\Omega \in$ $L^{\infty}\left(\mathbb{S}^{n-1}\right)$.
Let $1 \leq p<\infty$ and let $\varphi$ be a positive measurable function on $\mathbb{R}^{n} \times(0, \infty)$ and let $\omega$ be a nonnegative measurable function on $\mathbb{R}^{n}$. Following [12], we denote by $M_{\varphi}^{p}\left(\omega, \mathbb{R}^{n}\right)$ the generalized weighted Morrey space, the space of all functions $f \in L_{\text {loc }}^{p}\left(\omega, \mathbb{R}^{n}\right)$ with finite norm

$$
\begin{aligned}
& \|f\|_{M_{\varphi}^{p}\left(w, \mathbb{R}^{n}\right)} \\
& \quad=\sup _{x \in \mathbb{R}^{n}, r>0} \frac{1}{\varphi(x, r)}\left(\frac{1}{w(B(x, r))}\|f\|_{L^{p}(\omega, B(x, r))}^{p}\right)^{1 / p},
\end{aligned}
$$

where

$$
\|f\|_{L^{p}(\omega, B(x, r))}=\int_{B(x, r)}|f(y)|^{p} w(y) d y .
$$

If $\omega=1$ and $\varphi(x, r)=r^{(\lambda-n) / p}$ with $0 \leq \lambda \leq n$, then $M_{\varphi}^{p}\left(\omega, \mathbb{R}^{n}\right)=L^{p, \lambda}\left(\mathbb{R}^{n}\right)$ is the classical Morrey space; if $\varphi(x, r)=$ $\omega(B(x, r))^{(\kappa-1) / p}, 0<\kappa<1$, then $M_{\varphi}^{p}\left(\omega, \mathbb{R}^{n}\right)=L^{p, \kappa}\left(\omega, \mathbb{R}^{n}\right)$ is the weighted Morrey space; if $\omega=1, \varphi(x, r)^{p}=\Phi(r) r^{-n}$, then $M_{\varphi}^{p}\left(\omega, \mathbb{R}^{n}\right)=L^{p, \Phi}\left(\mathbb{R}^{n}\right)$. If $\omega=1$, then $M_{\varphi}^{p}\left(\omega, \mathbb{R}^{n}\right)=M_{\varphi}^{p}\left(\mathbb{R}^{n}\right)$.

The purpose of this paper is to discuss the boundedness properties of Marcinkiewicz integrals with rough kernel and their commutators on the generalized weighted Morrey spaces $M_{\varphi}^{p}\left(\omega, \mathbb{R}^{n}\right)$. Our main results can be formulated as follows.

Theorem 3. Suppose that $\Omega \in L^{q}\left(\mathbb{S}^{n-1}\right)$ with $1<q \leq \infty$. Let $q^{\prime}<p<\infty, \omega \in A_{p / q^{\prime}}$, and $\left(\varphi_{1}, \varphi_{2}\right)$ satisfy the condition

$$
\int_{s}^{\infty} \frac{\operatorname{ess}^{\inf _{r<t<\infty}} \varphi_{1}(x, t) \omega(B(x, t))^{1 / p}}{\omega(B(x, r))^{1 / p}} \frac{d r}{r} \leq C \varphi_{2}(x, s),
$$

where $C$ does not depend on $x$ or $r$. Then there is a constant $C>0$ independent of $f$ such that

$$
\left\|\mu_{\Omega} f\right\|_{M_{\varphi_{2}}^{p}\left(\omega, \mathbb{R}^{n}\right)} \leq C\|f\|_{M_{\varphi_{1}}^{p}\left(\omega, \mathbb{R}^{n}\right)} .
$$

Theorem 4. Suppose that $\Omega \in L^{q}\left(\mathbb{S}^{n-1}\right)$ with $1<q \leq \infty$. Let $q^{\prime}<p<\infty, \omega \in A_{p / q^{\prime}}$, and $\left(\varphi_{1}, \varphi_{2}\right)$ satisfy the condition

$$
\begin{aligned}
& \int_{s}^{\infty}\left(1+\ln \frac{r}{s}\right) \frac{\operatorname{ess~inf}_{r<t<\infty} \varphi_{1}(x, t) \omega(B(x, t))^{1 / p}}{\omega(B(x, r))^{1 / p}} \frac{d r}{r} \\
& \quad \leq C \varphi_{2}(x, s) .
\end{aligned}
$$

If $b \in B M O\left(\mathbb{R}^{n}\right)$, then there is a constant $C>0$ independent of $f$ such that

$$
\left\|\left[b, \mu_{\Omega}\right] f\right\|_{M_{\varphi_{2}}^{p}\left(\omega, \mathbb{R}^{n}\right)} \leq C\|b\|_{*}\|f\|_{M_{\varphi_{1}}^{p}\left(\omega, \mathbb{R}^{n}\right)} .
$$

Corollary 5. Let $\Omega \in L^{q}\left(\mathbb{S}^{n-1}\right)$ with $1<q \leq \infty$ and $q^{\prime}<p<$ $\infty$.

(i) Suppose $\left(\varphi_{1}, \varphi_{2}\right)$ satisfy the condition

$$
\int_{s}^{\infty} \frac{\operatorname{ess~inf}_{r<t<\infty} \varphi_{1}(x, t) t^{n / p}}{r^{1+(n / p)}} d r \leq C \varphi_{2}(x, s) .
$$


Then there is a constant $C>0$ independent of $f$ such that

$$
\left\|\mu_{\Omega} f\right\|_{M_{\varphi_{2}}^{p}\left(\mathbb{R}^{n}\right)} \leq C\|f\|_{M_{\varphi_{2}}^{p}\left(\mathbb{R}^{n}\right)} .
$$

(ii) Suppose $\left(\varphi_{1}, \varphi_{2}\right)$ satisfy the condition

$$
\int_{s}^{\infty}\left(1+\ln \frac{r}{s}\right) \frac{\operatorname{ess~inf}_{r<t<\infty} \varphi_{1}(x, t) t^{n / p}}{r^{1+(n / p)}} d r \leq C \varphi_{2}(x, s),
$$

and $b \in B M O\left(\mathbb{R}^{n}\right)$. Then there is a constant $C>0$ independent of $f$ such that

$$
\left\|\left[b, \mu_{\Omega}\right] f\right\|_{M_{\varphi_{2}}^{p}\left(\mathbb{R}^{n}\right)} \leq C\|b\|_{*}\|f\|_{M_{\varphi_{2}}^{p}\left(\mathbb{R}^{n}\right)} .
$$

Remark 6. (i) Let $\varphi_{1}(x, t)=\varphi_{2}(x, t)=\omega(B(x, t))^{(\kappa-1) / p}, 0<$ $\kappa<1$ and $w \in A_{p}\left(\mathbb{R}^{n}\right)(1 \leq p<\infty)$; then $\left(\varphi_{1}, \varphi_{2}\right)$ satisfy conditions (13) and (15).

(ii) Let $\varphi_{1}(x, t)=\varphi_{2}(x, t)=\left(\Phi(t) t^{-n}\right)^{1 / p}$. If $1 \leq D(\Phi) \leq$ $2^{n}$, then $\left(\varphi_{1}, \varphi_{2}\right)$ also satisfy conditions (13) and (15).

We verify only (i). (ii) can be verified similarly. In fact, from (36) in Section 2 we have constant $\delta>0$ such that

$$
\omega\left(B\left(x, 2^{k} s\right)\right) \geq C 2^{\delta} \omega(B(x, s)) .
$$

Then

$$
\begin{aligned}
& \int_{s}^{\infty} \frac{\operatorname{ess~inf}_{r<t<\infty} \varphi_{1}(x, t) \omega(B(x, t))^{1 / p}}{\omega(B(x, r))^{1 / p}} \frac{d r}{r} \\
& \leq \int_{s}^{\infty}\left(1+\ln \frac{r}{s}\right) \frac{\operatorname{ess~inf}_{r<t<\infty} \varphi_{1}(x, t) \omega(B(x, t))^{1 / p}}{\omega(B(x, r))^{1 / p}} \frac{d r}{r} \\
& \quad=\int_{s}^{\infty}\left(1+\ln \frac{r}{s}\right) \omega(B(x, r))^{(k-1) / p} \frac{d r}{r} \\
& \leq \sum_{k=0}^{\infty}(1+k) \int_{2^{k} s}^{2^{k+1} s} \omega(B(x, r))^{(k-1) / p} \frac{d r}{r} \\
& \leq C \sum_{k=0}^{\infty}(1+k) \omega\left(B\left(x, 2^{k+1} s\right)\right)^{(k-1) / p} \\
& \leq C \sum_{k=0}^{\infty}(1+k) 2^{-(k+1) \delta((1-\kappa) / p)} \omega(B(x, s))^{(k-1) / p} \\
& \leq C \omega(B(x, s))^{(k-1) / p}=C \varphi_{2}(x, s) .
\end{aligned}
$$

Corollary 7. Suppose that $\Omega \in L^{q}\left(\mathbb{S}^{n-1}\right)$ with $1<q \leq \infty$ and $0<\kappa<1$. If $q^{\prime}<p<\infty, \omega \in A_{p / q^{\prime}}$, then there is a constant $C>0$ independent of $f$ such that

$$
\left\|\mu_{\Omega} f\right\|_{L^{p, \kappa}\left(\omega, \mathbb{R}^{n}\right)} \leq C\|f\|_{L^{p, \kappa}\left(\omega, \mathbb{R}^{n}\right)} .
$$

If $q^{\prime}<p<\infty, \omega \in A_{p / q^{\prime}}$, and $b \in B M O\left(\mathbb{R}^{n}\right)$, then there is a constant $C>0$ independent of $f$ such that

$$
\left\|\left[b, \mu_{\Omega}\right] f\right\|_{L^{p, \kappa}\left(\omega, \mathbb{R}^{n}\right)} \leq C\|b\|_{*}\|f\|_{L^{p, \kappa}\left(\omega, \mathbb{R}^{n}\right)} .
$$

Corollary 8. Suppose that $\Omega \in L^{q}\left(\mathbb{S}^{n-1}\right)$ with $1<q \leq \infty$. If $q^{\prime}<p<\infty, 1 \leq D(\Phi) \leq 2^{n}$, then there is a constant $C>0$ independent of $f$ such that

$$
\left\|\mu_{\Omega} f\right\|_{L^{p, \Phi}\left(\mathbb{R}^{n}\right)} \leq C\|f\|_{L^{p, \Phi}\left(\mathbb{R}^{n}\right)} .
$$

If $q^{\prime}<p<\infty, 1 \leq D(\Phi) \leq 2^{n}$, and $b \in B M O\left(\mathbb{R}^{n}\right)$, then there is a constant $C>0$ independent of $f$ such that

$$
\left\|\left[b, \mu_{\Omega}\right] f\right\|_{L^{p, \Phi}\left(\mathbb{R}^{n}\right)} \leq C\|b\|_{*}\|f\|_{L^{p, \Phi}\left(\mathbb{R}^{n}\right)} .
$$

\section{Some Preliminaries}

We begin with some properties of $A_{p}$ weights which play a great role in the proofs of our main results.

A weight $\omega$ is a nonnegative, locally integrable function on $\mathbb{R}^{n}$. Let $B=B\left(x_{0}, r_{B}\right)$ denote the ball with the center $x_{0}$ and radius $r_{B}$. For a given weight function $\omega$ and a measurable set $E$, we also denote the Lebesgue measure of $E$ by $|E|$ and set weighted measure $\omega(E)=\int_{E} \omega(x) d x$. For any given weight function $\omega$ on $\mathbb{R}^{n}, X \subseteq \mathbb{R}^{n}$, and $0<p<\infty$, denote by $L^{p}(\omega, X)$ the space of all function $f$ satisfying

$$
\|f\|_{L^{p}(\omega, X)}=\left(\int_{X}|f(x)|^{p} \omega(x) d x\right)^{1 / p}<\infty .
$$

A weight $\omega$ is said to belong to $A_{p}\left(\mathbb{R}^{n}\right)$ for $1<p<\infty$, if there exists a constant

$$
\left(\frac{1}{|B|} \int_{B} \omega(x) d x\right)\left(\frac{1}{|B|} \int_{B} \omega(x)^{1-p^{\prime}} d x\right)^{p-1} \leq C,
$$

where $p^{\prime}$ is the dual of $p$ such that $1 / p+1 / p^{\prime}=1$. The class $A_{1}\left(\mathbb{R}^{n}\right)$ is defined by

$$
\begin{array}{r}
\frac{1}{|B|} \int_{B} w(y) d y \leq C \cdot \underset{x \in B}{\operatorname{ess} \inf } w(x) \\
\text { for every ball } B \subset \mathbb{R}^{n} .
\end{array}
$$

A weight $\omega$ is said to belong to $A_{\infty}\left(\mathbb{R}^{n}\right)$ if there are positive numbers $C$ and $\delta$ so that

$$
\frac{\omega(E)}{\omega(B)} \leq C\left(\frac{|E|}{|B|}\right)^{\delta}
$$

for all balls $B$ and all measurable $E \subset B$. It is well known that

$$
A_{\infty}\left(\mathbb{R}^{n}\right)=\bigcup_{1 \leq p<\infty} A_{p}\left(\mathbb{R}^{n}\right) .
$$

By (28), we have

$$
\left(\omega^{-\left(p^{\prime} / p\right)}(B)\right)^{1 / p^{\prime}}=\left\|\omega^{-(1 / p)}\right\|_{L^{p^{\prime}}(B)} \leq C|B|(\omega(B))^{-(1 / p)}
$$

for $1<p<\infty$. Note that

$$
(\underset{x \in E}{\operatorname{ess} \inf f(x)})^{-1}=\underset{x \in E}{\operatorname{ess} \sup } \frac{1}{f(x)}
$$


is true for any real-valued nonnegative function $f$ and measurable on $E$ (see [13] page 143) and (29); we get

$$
\begin{aligned}
\left\|\omega^{-1}\right\|_{L^{\infty}(B)} & =\underset{x \in B}{\operatorname{ess} \sup } \frac{1}{w(x)} \\
& =\frac{1}{\underset{x \in B}{\operatorname{ess} \inf } w(x)} \leq C|B| \omega(B)^{-1} .
\end{aligned}
$$

The classical $A_{p}\left(\mathbb{R}^{n}\right)$ weight theory was first introduced by Muckenhoupt in the study of weighted $L^{p}$-boundedness of Hardy-Littlewood maximal function in [14].

Lemma 9 (see $[14,15])$. Suppose $\omega \in A_{p}\left(\mathbb{R}^{n}\right)$ and the following statements hold.

(i) For any $1 \leq p<\infty$, there is a positive number $C$ such that

$$
\frac{\omega\left(B_{k}\right)}{\omega\left(B_{j}\right)} \leq C 2^{n p(k-j)} \quad \text { for } k>j
$$

(ii) For any $1 \leq p<\infty$, there are positive numbers $C$ and $\delta$ such that

$$
\frac{\omega\left(B_{k}\right)}{\omega\left(B_{j}\right)} \geq C 2^{\delta(k-j)} \quad \text { for } k>j .
$$

(iii) For any $1<p<\infty$, one has $\omega^{1-p^{\prime}} \in A_{p^{\prime}}\left(\mathbb{R}^{n}\right)$.

Following [16], a locally integrable function $b$ is said to be in $B M O\left(\mathbb{R}^{n}\right)$ if

$$
\sup _{B \subset \mathbb{R}^{n}} \frac{1}{|B|} \int_{B}\left|b(x)-b_{B}\right| d x=\|b\|_{*}<\infty,
$$

where

$$
b_{B}=\frac{1}{|B|} \int_{B} b(y) d y .
$$

Lemma 10 (see [12]). Suppose $\omega \in A_{\infty}\left(\mathbb{R}^{n}\right)$ and $b \in$ $B M O\left(\mathbb{R}^{n}\right)$. Then, for any $1 \leq p<\infty$ and $r_{1}, r_{2}>0$, one has

$$
\begin{aligned}
& \left(\frac{1}{\omega\left(B\left(x_{0}, r_{1}\right)\right)} \int_{B\left(x_{0}, r_{1}\right)}\left|b(x)-b_{B\left(x_{0}, r_{2}\right)}\right|^{p} \omega(x) d x\right)^{1 / p} \\
& \quad \leq C\left(1+\left|\ln \frac{r_{1}}{r_{2}}\right|\right)\|b\|_{*} .
\end{aligned}
$$

\section{Proof of Theorem 3}

We first prove the following conclusions.
Theorem 11. Suppose that $\Omega \in L^{q}\left(\mathbb{S}^{n-1}\right), 1<q \leq \infty$. Then, for every $q^{\prime}<p<\infty, \omega \in A_{p / q^{\prime}}$, there is a constant $C$ independent of $f$ such that

$$
\begin{aligned}
&\left\|\mu_{\Omega}(f)\right\|_{L^{p}\left(\omega, B\left(x_{0}, s\right)\right)} \\
& \leq C \omega\left(B\left(x_{0}, s\right)\right)^{1 / p} \\
& \quad \cdot \int_{2 s}^{\infty}\|f\|_{L^{p}\left(\omega, B\left(x_{0}, r\right)\right)} \omega\left(B\left(x_{0}, r\right)\right)^{-(1 / p)} \frac{d r}{r} .
\end{aligned}
$$

Proof. We represent $f$ as $f=f_{1}+f_{2}$, where $f_{1}(y)=$ $f(y) \chi_{B\left(x_{0}, 2 s\right)}(y), \chi_{B\left(x_{0}, 2 s\right)}$ denotes the characteristic function of $B\left(x_{0}, 2 s\right)$. Then

$$
\begin{aligned}
& \left\|\mu_{\Omega}(f)\right\|_{L^{p}\left(\omega, B\left(x_{0}, s\right)\right)} \\
& \quad \leq\left\|\mu_{\Omega}\left(f_{1}\right)\right\|_{L^{p}\left(\omega, B\left(x_{0}, s\right)\right)}+\left\|\mu_{\Omega}\left(f_{2}\right)\right\|_{L^{p}\left(\omega, B\left(x_{0}, s\right)\right)} .
\end{aligned}
$$

Since $f_{1} \in L^{p}\left(\omega, \mathbb{R}^{n}\right)$ and from the boundedness of $\mu_{\Omega}$ on $L^{p}\left(\omega, \mathbb{R}^{n}\right)\left(q^{\prime}<p<\infty\right)$ (Theorem 1$)$ it follows that

$$
\begin{aligned}
\left\|\mu_{\Omega}\left(f_{1}\right)\right\|_{L^{p}\left(\omega, B\left(x_{0}, s\right)\right)} & \leq\left\|\mu_{\Omega}\left(f_{1}\right)\right\|_{L^{p}\left(\omega, \mathbb{R}^{n}\right)} \\
& \leq C\left\|f_{1}\right\|_{L^{p}\left(\omega, \mathbb{R}^{n}\right)}=C\|f\|_{L^{p}\left(\omega, B\left(x_{0}, 2 s\right)\right)} .
\end{aligned}
$$

By Hölder inequality,

$$
\left|B\left(x_{0}, s\right)\right| \leq C \omega\left(B\left(x_{0}, s\right)\right)^{1 / p}\left\|\omega^{-(1 / p)}\right\|_{L^{p^{\prime}\left(B\left(x_{0}, s\right)\right)}} .
$$

Then, for $q^{\prime}<p<\infty$,

$$
\begin{aligned}
& \left\|\mu_{\Omega}\left(f_{1}\right)\right\|_{L^{p}\left(\omega, B\left(x_{0}, s\right)\right)} \\
& \leq C\left|B\left(x_{0}, s\right)\right|\|f\|_{L^{p}\left(\omega, B\left(x_{0}, 2 s\right)\right)} \int_{2 s}^{\infty} \frac{d r}{r^{n+1}} \\
& \leq C\left|B\left(x_{0}, s\right)\right| \int_{2 s}^{\infty}\|f\|_{L^{p}\left(\omega, B\left(x_{0}, r\right)\right)} \frac{d r}{r^{n+1}} \\
& \leq C \omega\left(B\left(x_{0}, s\right)\right)^{1 / p}\left\|\omega^{-(1 / p)}\right\|_{L^{p^{\prime}}\left(B\left(x_{0}, s\right)\right)} \\
& \quad \cdot \int_{2 s}^{\infty}\|f\|_{L^{p}\left(\omega, B\left(x_{0}, r\right)\right)} \frac{d r}{r^{n+1}} \\
& \leq C \omega\left(B\left(x_{0}, s\right)\right)^{1 / p} \\
& \quad \cdot \int_{2 s}^{\infty}\|f\|_{L^{p}\left(\omega, B\left(x_{0}, r\right)\right)}\left\|\omega^{-(1 / p)}\right\|_{L^{p^{\prime}}\left(B\left(x_{0}, r\right)\right)} \frac{d r}{r^{n+1}} .
\end{aligned}
$$

By (32), we get

$$
\begin{aligned}
& \left\|\mu_{\Omega}\left(f_{1}\right)\right\|_{L^{p}\left(\omega, B\left(x_{0}, s\right)\right)} \leq C \omega\left(B\left(x_{0}, s\right)\right)^{1 / p} \\
& \quad \cdot \int_{2 s}^{\infty}\|f\|_{L^{p}\left(\omega, B\left(x_{0}, r\right)\right)} \omega\left(B\left(x_{0}, r\right)\right)^{-(1 / p)} \frac{d r}{r} .
\end{aligned}
$$

To estimate $\left\|\mu_{\Omega}\left(f_{2}\right)\right\|_{L^{p}\left(\omega, B\left(x_{0}, s\right)\right)}$, observe that when $x \in$ $B\left(x_{0}, s\right)$ and $y \in B\left(x_{0}, 2^{j+1} s\right) \backslash B\left(x_{0}, 2^{j} s\right)(j \geq 1)$,

$$
t \geq|x-y| \geq\left|y-x_{0}\right|-\left|x-x_{0}\right| \geq 2^{j-1} s .
$$


Therefore, by Minkowski’s inequality, we have

$$
\begin{aligned}
& \mu_{\Omega}\left(f_{2}\right)(x) \\
& =\left(\int_{0}^{\infty} \mid \int_{B\left(x_{0}, 2 s\right)^{c} \cap\{y:|x-y| \leq t\}} \frac{\Omega(x-y)}{\left.\left.|x-y|^{n-1} f(y) d y\right|^{2} \frac{d t}{t^{3}}\right)^{1 / 2}}\right. \\
& =\left(\int_{0}^{\infty} \mid \sum_{j=1}^{\infty} \int_{\left(B\left(x_{0}, 2^{j+1} l\right) \backslash B\left(x_{0}, 2^{j} l\right)\right)^{c} \cap\{y:|x-y| \leq t\}} \frac{\Omega(x-y)}{|x-y|^{n-1}}\right. \\
& \leq C \sum_{j=1}^{\infty}\left(\left.\int_{B\left(x_{0}, 2^{j+1} s\right) \backslash B\left(x_{0}, 2^{j} s\right)} \frac{|\Omega(x-y)|}{\left.|x-y|^{n-1}|f(y)| d y\right)}\right|^{2} \frac{d t}{t^{3}}\right)^{1 / 2} \\
& \cdot\left(\int_{2^{j-1} s}^{\infty} \frac{d t}{t^{3}}\right)^{1 / 2} \sum_{j=1}^{\infty}\left(2^{j+1} s\right)^{-1} \int_{B\left(x_{0}, 2^{j+1} s\right) \backslash B\left(x_{0}, 2^{j} s\right)} \frac{|\Omega(x-y)|}{|x-y|^{n-1}}|f(y)| d y .
\end{aligned}
$$

When $\Omega \in L^{\infty}\left(\mathbb{S}^{n-1}\right)$, then by assumption we have $\omega \epsilon$ $A_{p}, 1<p<\infty$. It follows from the Hölder inequality and (32) that

$$
\begin{aligned}
& \sup _{x \in B\left(x_{0}, s\right)}\left|\mu_{\Omega}\left(f_{2}\right)(x)\right| \\
& \leq C\|\Omega\|_{L^{\infty}\left(\mathbb{S}^{n-1}\right)} \sum_{j=1}^{\infty}\left(2^{j+1} s\right)^{-n} \int_{B\left(x_{0}, 2^{j+1} s\right)}|f(y)| d y \\
& \leq C \sum_{j=1}^{\infty}\left(2^{j+1} s\right)^{-n}\|f\|_{L^{p}\left(\omega, B\left(x_{0}, 2^{j+1} s\right)\right)} \\
& \leq C \sum_{j=1}^{\infty} \int_{2^{j+1} s}^{2^{j+2} s}\left(2^{j+1} s\right)^{-(1+n)}\|f\|_{L^{p}\left(\omega, B\left(x_{0}, r\right)\right)}{ }^{-(1 / p)}\left\|_{L^{p^{\prime}}\left(B\left(x_{0}, 2^{j+1} s\right)\right)} \cdot\right\| \omega^{-(1 / p)} \|_{L^{p^{\prime}}\left(B\left(x_{0}, r\right)\right)} d r \\
& \leq C \int_{2 s}^{\infty}\|f\|_{L^{p}\left(\omega, B\left(x_{0}, r\right)\right)}\left\|\omega^{-(1 / p)}\right\|_{L^{p^{\prime}}\left(B\left(x_{0}, r\right)\right)} \frac{d r}{r^{1+n}} \\
& \leq C \int_{2 s}^{\infty}\|f\|_{L^{p}\left(\omega, B\left(x_{0}, r\right)\right)} \omega\left(B\left(x_{0}, r\right)\right)^{-(1 / p)} \frac{d r}{r} .
\end{aligned}
$$

Then

$$
\begin{aligned}
& \left\|\mu_{\Omega}\left(f_{2}\right)\right\|_{L^{p}\left(\omega, B\left(x_{0}, s\right)\right)} \\
& \leq C \omega\left(B\left(x_{0}, s\right)\right)^{1 / p} \int_{2 s}^{\infty}\|f\|_{L^{p}\left(\omega, B\left(x_{0}, r\right)\right)} \\
& \cdot \omega\left(B\left(x_{0}, r\right)\right)^{-(1 / p)} \frac{d r}{r} .
\end{aligned}
$$

When $\Omega \in L^{q}\left(\mathbb{S}^{n-1}\right), 1<q<\infty$. It follows from the Hölder inequality that

$$
\begin{aligned}
\int_{B\left(x_{0}, 2^{j+1} s\right) \backslash B\left(x_{0}, 2^{j} s\right)} \frac{|\Omega(x-y)|}{|x-y|^{n-1}}|f(y)| d y \\
\leq C\left(\int_{B\left(x_{0}, 2^{j+1} s\right) \backslash B\left(x_{0}, 2^{j} s\right)}\left|\Omega\left((x-y)^{\prime}\right)\right|^{q} d y\right)^{1 / q} \\
\quad\left(\int_{B\left(x_{0}, 2^{j+1} s\right) \backslash B\left(x_{0}, 2^{j} s\right)} \frac{|f(y)|^{q^{\prime}}}{|x-y|^{(n-1) q^{\prime}}} d y\right)^{1 / q^{\prime}} .
\end{aligned}
$$

When $x \in B\left(x_{0}, s\right)$ and $y \in B\left(x_{0}, 2^{j+1} s\right) \backslash B\left(x_{0}, 2^{j} s\right)$, then by a direct calculation we can see that $2^{j-1} s \leq|y-x|<2^{j+1} s$. Hence

$$
\begin{aligned}
& \left(\int_{B\left(x_{0}, 2^{j+1} s\right) \backslash B\left(x_{0}, 2^{j} s\right)}\left|\Omega\left((x-y)^{\prime}\right)\right|^{q} d y\right)^{1 / q} \\
& \leq C\|\Omega\|_{L^{q}\left(\mathbb{S}^{n-1}\right)}\left|B\left(x_{0}, 2^{j+1} s\right)\right|^{1 / q} .
\end{aligned}
$$

We also note that if $x \in B\left(x_{0}, s\right), y \in B\left(x_{0}, 2 s\right)^{c}$, then $|y-x| \sim$ $\left|y-x_{0}\right|$. Consequently

$$
\begin{aligned}
& \left(\int_{B\left(x_{0}, 2^{j+1} s\right) \backslash B\left(x_{0}, 2^{j} s\right)} \frac{|f(y)|^{q^{\prime}}}{|x-y|^{(n-1) q^{\prime}}} d y\right)^{1 / q^{\prime}} \\
& \leq\left(2^{j+1} s\right)^{1-n}\left(\int_{B\left(x_{0}, 2^{j+1} s\right)}|f(y)|^{q^{\prime}} d y\right)^{1 / q^{\prime}} .
\end{aligned}
$$

It follows from (47), (50), (51), and (52) that

$$
\begin{aligned}
& \sup _{x \in B\left(x_{0}, s\right)}\left|\mu_{\Omega}\left(f_{2}\right)(x)\right| \\
& \leq C \sum_{j=1}^{\infty}\left(2^{j+1} s\right)^{-\left(n / q^{\prime}\right)}\left(\int_{B\left(x_{0}, 2^{j+1} s\right)}|f(y)|^{q^{\prime}} d y\right)^{1 / q^{\prime}} .
\end{aligned}
$$

Since $q^{\prime}<p<\infty, v=p / q^{\prime}>1$. Thus from the Hölder inequality we get that

$$
\begin{gathered}
\sup _{x \in B\left(x_{0}, s\right)}\left|\mu_{\Omega}\left(f_{2}\right)(x)\right| \\
\leq C \sum_{j=1}^{\infty}\left(2^{j+1} s\right)^{-n / q^{\prime}}\|f\|_{L^{p}\left(\omega, B\left(x_{0}, 2^{j+1} s\right)\right)} \\
\cdot\left\|\omega^{-(1 / p)}\right\|_{L^{q^{\prime} v^{\prime}}\left(B\left(x_{0}, 2^{j+1} s\right)\right)} \\
\leq C \sum_{j=1}^{\infty} \int_{2^{j+1} s}^{2^{j+2} s}\left(2^{j+1} s\right)^{-\left(1+n / q^{\prime}\right)} \\
\leq \int_{2 s}^{\infty}\|f\|_{L^{p}\left(\omega, B\left(x_{0}, r\right)\right)} \cdot\|f\|_{L^{p}\left(\omega, B\left(x_{0}, r\right)\right)}\left\|\omega^{-(1 / p)}\right\|_{L^{q^{\prime} v^{\prime}}\left(B\left(x_{0}, r\right)\right)} d r \\
\cdot\left\|\omega^{-(1 / p)}\right\|_{L^{q^{\prime} v^{\prime}}\left(B\left(x_{0}, r\right)\right)} \frac{d r}{r^{1+n / q^{\prime}}} .
\end{gathered}
$$


Note that $\omega \in A_{\nu}$. It follows from the $A_{v}$ condition that

$$
\omega\left(B\left(x_{0}, r\right)\right)\left(\omega^{1-\nu^{\prime}}\left(B\left(x_{0}, r\right)\right)\right)^{\nu-1} \leq C .
$$

That is,

$$
\left\|\omega^{-(1 / p)}\right\|_{L^{q^{\prime} v^{\prime}}\left(B\left(x_{0}, r\right)\right)} \leq r^{n / q^{\prime}} \omega\left(B\left(x_{0}, r\right)\right)^{-(1 / p)} .
$$

Then

$$
\begin{aligned}
& \sup _{x \in B\left(x_{0}, s\right)}\left|\mu_{\Omega}\left(f_{2}\right)(x)\right| \\
& \quad \leq C \int_{2 s}^{\infty}\|f\|_{L^{p}\left(\omega, B\left(x_{0}, r\right)\right)} \omega\left(B\left(x_{0}, r\right)\right)^{-(1 / p)} \frac{d r}{r} .
\end{aligned}
$$

Therefore

$$
\begin{aligned}
& \left\|\mu_{\Omega}\left(f_{2}\right)\right\|_{L^{p}\left(\omega, B\left(x_{0}, s\right)\right)} \\
& \leq C \omega\left(B\left(x_{0}, s\right)\right)^{1 / p} \\
& \quad \cdot \int_{2 s}^{\infty}\|f\|_{L^{p}\left(\omega, B\left(x_{0}, r\right)\right)} \omega\left(B\left(x_{0}, r\right)\right)^{-(1 / p)} \frac{d r}{r} .
\end{aligned}
$$

Combining (45), (49), and (58), the proof of Theorem 11 is completed.

Proof of Theorem 3. Since $f \in M_{\varphi_{1}}^{p}\left(w, \mathbb{R}^{n}\right)$, from (33) and the fact $\|f\|_{L^{p}\left(\omega, B\left(x_{0}, r\right)\right)}$ is a nondecreasing function of $r$, we get

$$
\begin{gathered}
\frac{\|f\|_{L^{p}\left(\omega, B\left(x_{0}, r\right)\right)}}{\text { ess } \inf _{0<r<t<\infty} \varphi_{1}\left(x_{0}, t\right) \omega\left(B\left(x_{0}, t\right)\right)^{1 / p}} \\
\leq \underset{0<r<t<\infty}{\operatorname{ess} \sup } \frac{\|f\|_{L_{1}^{p}\left(\omega, B\left(x_{0}, r\right)\right)}\left(x_{0}, t\right) \omega\left(B\left(x_{0}, t\right)\right)^{1 / p}}{\| \operatorname{ess}_{0<t<\infty} \frac{\|f\|_{L^{p}\left(\omega, B\left(x_{0}, t\right)\right)}}{\varphi_{1}\left(x_{0}, t\right) \omega\left(B\left(x_{0}, t\right)\right)^{1 / p}}} \\
\leq\|f\|_{M_{\varphi_{1}}^{p}\left(w, \mathbb{R}^{n}\right)} \cdot
\end{gathered}
$$

For $q^{\prime}<p<\infty$, since $\left(\varphi_{1}, \varphi_{2}\right)$ satisfy (13), we have

$$
\begin{gathered}
\int_{s}^{\infty}\|f\|_{L^{p}\left(\omega, B\left(x_{0}, r\right)\right)} \omega\left(B\left(x_{0}, r\right)\right)^{-(1 / p)} \frac{d r}{r} \\
\leq \int_{s}^{\infty} \frac{\|f\|_{L^{p}\left(\omega, B\left(x_{0}, r\right)\right)} \frac{\operatorname{ess~inf}_{r<t<\infty} \varphi_{1}\left(x_{0}, t\right) \omega\left(B\left(x_{0}, t\right)\right)^{1 / p}}{\omega}\left(B\left(x_{0}, r\right)\right)^{1 / p}}{\cdot \frac{\operatorname{ess~inf}_{r<t<\infty} \varphi_{1}\left(x_{0}, t\right) \omega\left(B\left(x_{0}, t\right)\right)^{1 / p}}{d r}} \\
\leq C\|f\|_{M_{\varphi_{1}}^{p}\left(w, \mathbb{R}^{n}\right)} \\
\cdot \int_{s}^{\infty} \frac{\operatorname{ess}_{\inf _{r<t<\infty}} \varphi_{1}\left(x_{0}, t\right) \omega\left(B\left(x_{0}, t\right)\right)^{1 / p}}{\omega\left(B\left(x_{0}, r\right)\right)^{1 / p}} \frac{d r}{r} \\
\leq C\|f\|_{M_{\varphi_{1}}^{p}\left(w, \mathbb{R}^{n}\right)} \varphi_{2}\left(x_{0}, s\right) .
\end{gathered}
$$

Then by (40), we get

$$
\begin{aligned}
& \left\|\mu_{\Omega}(f)\right\|_{M_{\varphi_{2}}^{p}\left(w, \mathbb{R}^{n}\right)} \\
& \leq C \sup _{x_{0} \in \mathbb{R}^{n}, s>0} \frac{1}{\varphi_{2}\left(x_{0}, s\right)} \\
& \quad \cdot\left(\frac{1}{w\left(B\left(x_{0}, s\right)\right)} \int_{B\left(x_{0}, s\right)}\left|\mu_{\Omega}(f)(y)\right|^{p} w(y) d y\right)^{1 / p} \\
& \leq C \sup _{x_{0} \in \mathbb{R}^{n}, s>0} \frac{1}{\varphi_{2}\left(x_{0}, s\right)} \\
& \quad \cdot \int_{s}^{\infty}\|f\|_{L^{p}\left(\omega, B\left(x_{0}, r\right)\right)} \omega\left(B\left(x_{0}, r\right)\right)^{-(1 / p)} \frac{d r}{r} \\
& \leq C\|f\|_{M_{\varphi_{1}}^{p}\left(w, \mathbb{R}^{n}\right)} \cdot
\end{aligned}
$$

This completes the proof of Theorem 3.

\section{Proof of Theorem 4}

As in the proof of Theorem 3, it suffices to prove the following result.

Theorem 12. Assume that $\Omega \in L^{q}\left(\mathbb{S}^{n-1}\right)$ with $1<q \leq \infty$ and $b \in B M O\left(\mathbb{R}^{n}\right)$. Then, for every $q^{\prime}<p<\infty, \omega \in A_{p / q^{\prime}}$, there is a constant $C$ independent of $f$ such that

$$
\begin{aligned}
\left\|\left[b, \mu_{\Omega}\right](f)\right\|_{L^{p}\left(\omega, B\left(x_{0}, s\right)\right)} & \\
\leq & C\|b\|_{*} \omega\left(B\left(x_{0}, s\right)\right)^{1 / p} \\
& \quad \int_{2 s}^{\infty}\left(1+\ln \frac{r}{s}\right)\|f\|_{L^{p}\left(\omega, B\left(x_{0}, r\right)\right)} \omega\left(B\left(x_{0}, r\right)\right)^{-(1 / p)} \frac{d r}{r} .
\end{aligned}
$$

Proof. We represent $f$ as

$$
\begin{aligned}
f(y) & =f_{1}+f_{2}, \\
f_{1}(y) & =f(y) \chi_{B\left(x_{0}, 2 s\right)}(y), \\
f_{2}(y) & =f(y) \chi_{\left(B\left(x_{0}, 2 s\right)\right)^{c}}(y) .
\end{aligned}
$$

Then

$$
\begin{aligned}
\left\|\left[b, \mu_{\Omega}\right](f)\right\|_{L^{p}\left(\omega, B\left(x_{0}, s\right)\right)} \\
\leq\left\|\left[b, \mu_{\Omega}\right]\left(f_{1}\right)\right\|_{L^{p}\left(\omega, B\left(x_{0}, s\right)\right)} \\
\quad+\left\|\left[b, \mu_{\Omega}\right]\left(f_{2}\right)\right\|_{L^{p}\left(\omega, B\left(x_{0}, s\right)\right)} .
\end{aligned}
$$

By Theorem 2, we have

$$
\left\|\left[b, \mu_{\Omega}\right]\left(f_{1}\right)\right\|_{L^{p}\left(\omega, B\left(x_{0}, s\right)\right)} \leq C\|b\|_{*}\|f\|_{L^{p}\left(\omega, B\left(x_{0}, 2 s\right)\right)} .
$$


As the proof of (45), we get

$$
\begin{aligned}
\left\|\left[b, T_{\Omega}\right]\left(f_{1}\right)\right\|_{L^{p}\left(\omega, B\left(x_{0}, s\right)\right)} & \\
\leq & C\|b\|_{*} \omega\left(B\left(x_{0}, s\right)\right)^{1 / p} \\
& \cdot \int_{2 s}^{\infty}\|f\|_{L^{p}\left(\omega, B\left(x_{0}, r\right)\right)} \omega\left(B\left(x_{0}, r\right)\right)^{-(1 / p)} \frac{d r}{r} .
\end{aligned}
$$

We now turn to deal with the term $\left\|\left[b, \mu_{\Omega}\right]\left(f_{2}\right)\right\|_{L^{p}\left(\omega, B\left(x_{0}, s\right)\right)}$. For any given $x \in B\left(x_{0}, s\right)$, we have

$$
\begin{aligned}
& \left|\left[b, \mu_{\Omega}\right]\left(f_{2}\right)(x)\right| \\
& \leq C\left|b(x)-b_{B\left(x_{0}, s\right)}\right|\left|\mu_{\Omega}\left(f_{2}\right)(x)\right| \\
& \quad+C\left|\mu_{\Omega}\left(\left(b-b_{B\left(x_{0}, s\right)}\right) f_{2}\right)(x)\right| \\
& =I_{1}+I_{2} .
\end{aligned}
$$

By (48) and (57), for any $\Omega \in L^{q}\left(\mathbb{S}^{n-1}\right), 1<q \leq \infty$, we have

$$
\begin{aligned}
I_{1} \leq & C\left|b(y)-b_{B\left(x_{0}, s\right)}\right| \\
& \cdot \int_{2 s}^{\infty}\|f\|_{L^{p}\left(\omega, B\left(x_{0}, r\right)\right)} \omega\left(B\left(x_{0}, r\right)\right)^{-(1 / p)} \frac{d r}{r} .
\end{aligned}
$$

Then from Lemma 10 we get

$$
\begin{aligned}
\left\|I_{1}\right\|_{L^{p}\left(\omega, B\left(x_{0}, s\right)\right)} \leq & C\|b\|_{*} \omega\left(B\left(x_{0}, s\right)\right)^{1 / p} \\
& \cdot \int_{2 s}^{\infty}\|f\|_{L^{p}\left(\omega, B\left(x_{0}, r\right)\right)} \omega\left(B\left(x_{0}, r\right)\right)^{-(1 / p)} \frac{d r}{r} .
\end{aligned}
$$

When $\Omega \in L^{\infty}\left(\mathbb{S}^{n-1}\right)$, by assumption, we have $\omega \in A_{p}$, $1<p<\infty$. It follows from the Hölder inequality that

$$
\begin{aligned}
\sup _{x \in B\left(x_{0}, s\right)}\left|\mu_{\Omega}\left(\left(b-b_{B\left(x_{0}, s\right)}\right) f_{2}\right)(x)\right| \\
\leq C\|\Omega\|_{L^{\infty}\left(\mathbb{S}^{n-1}\right)} \sum_{j=1}^{\infty}\left(2^{j+1} s\right)^{-n} \\
\cdot \int_{B\left(x_{0}, 2^{j+1} s\right)}\left|b(y)-b_{B\left(x_{0}, s\right)}\right||f(y)| d y \\
\leq C \sum_{j=1}^{\infty}\left(2^{j+1} s\right)^{-n}\|f\|_{L^{p}\left(\omega, B\left(x_{0}, 2^{j+1} s\right)\right)} \\
\cdot\left\|b(\cdot)-b_{B\left(x_{0}, s\right)}\right\|_{L^{p^{\prime}}\left(\omega^{1-p^{\prime}}, B\left(x_{0}, 2^{j+1} s\right)\right)} \\
\leq C \sum_{j=1}^{\infty} \int_{2^{j+1} s}^{2^{j+2} s}\left(2^{j+1} s\right)^{-(1+n)}\|f\|_{L^{p}\left(\omega, B\left(x_{0}, r\right)\right)} \\
\quad\left\|b(\cdot)-b_{B\left(x_{0}, s\right)}\right\|_{L^{p^{\prime}}\left(\omega^{1-p^{\prime}}, B\left(x_{0}, r\right)\right)} d r \\
\leq C \int_{2 s}^{\infty}\|f\|_{L^{p}\left(\omega, B\left(x_{0}, r\right)\right)} \\
\quad\left\|b(\cdot)-b_{B\left(x_{0}, s\right)}\right\|_{L^{p^{\prime}}\left(\omega^{1-p^{\prime}}, B\left(x_{0}, r\right)\right)} \frac{d r}{r^{1+n}} .
\end{aligned}
$$

Set $s=p / q^{\prime}$. Since $\omega \in A_{s}$, from Lemma 9, we know $\omega^{1-s^{\prime}} \in$ $A_{s^{\prime}}$. By Hölder inequality

$$
\begin{gathered}
\left(\int_{B\left(x_{0}, 2^{j+1} s\right)}\left|b(y)-b_{B\left(x_{0}, s\right)}\right|^{q^{\prime}}|f(y)|^{q^{\prime}} d y\right)^{1 / q^{\prime}} \\
\leq C\|f\|_{L^{p}\left(\omega, B\left(x_{0}, 2^{j+1} s\right)\right)} \\
\cdot\left\|b(\cdot)-b_{B\left(x_{0}, s\right)}\right\|_{L^{q^{\prime} s^{\prime}}\left(\omega^{1-s^{\prime}}, B\left(x_{0}, 2^{j+1} s\right)\right)}
\end{gathered}
$$

Therefore,

$$
\begin{aligned}
& \left\|I_{2}\right\|_{L^{p}\left(\omega, B\left(x_{0}, s\right)\right)} \\
& \leq C\|b\|_{*} \omega\left(B\left(x_{0}, s\right)\right)^{1 / p} \\
& \quad \cdot \int_{2 s}^{\infty}\|f\|_{L^{p}\left(\omega, B\left(x_{0}, r\right)\right)} \omega\left(B\left(x_{0}, r\right)\right)^{-(1 / p)} \frac{d r}{r} .
\end{aligned}
$$
that

When $\Omega \in L^{q}\left(\mathbb{S}^{n-1}\right)$, it follows from (50), (51), and (52)

$$
\begin{aligned}
I_{2} \leq & C \sum_{j=1}^{\infty}\left(2^{j+1} s\right)^{-\left(n / q^{\prime}\right)} \\
& \cdot\left(\int_{B\left(x_{0}, 2^{j+1} s\right)}\left|\left(b(y)-b_{B\left(x_{0}, s\right)}\right) f(y)\right|^{q^{\prime}} d y\right)^{1 / q^{\prime}} .
\end{aligned}
$$


Consequently,

$$
\begin{gathered}
I_{2} \leq \sum_{j=1}^{\infty} \int_{2^{j+1} s}^{2^{j+2} s}\left(2^{j+1} s\right)^{-\left(1+\left(n / q^{\prime}\right)\right)} \\
\cdot\|f\|_{L^{p}\left(\omega, B\left(x_{0}, 2^{j+1} s\right)\right)} \\
\cdot\left\|b(\cdot)-b_{B\left(x_{0}, s\right)}\right\|_{L^{q^{\prime} s^{\prime}}\left(\omega^{1-s^{\prime}}, B\left(x_{0}, 2^{j+1} s\right)\right)} d r \\
\leq C \int_{2 s}^{\infty}\|f\|_{L^{p}\left(\omega, B\left(x_{0}, r\right)\right)} \\
\cdot\left\|b(\cdot)-b_{B\left(x_{0}, s\right)}\right\|_{L^{q^{\prime} s^{\prime}}\left(\omega^{1-s^{\prime}}, r\right)} \frac{d r}{r^{1+n / q^{\prime}}} .
\end{gathered}
$$

Since $\omega^{1-s^{\prime}} \in A_{s^{\prime}}$, from (32), we know

$$
\left(\omega^{1-s^{\prime}} B\left(x_{0}, r\right)\right)^{1 / q^{\prime} s^{\prime}} \leq C r^{n / q^{\prime}}\left(B\left(x_{0}, r\right)\right)^{-(1 / p)}
$$

Using Lemma 10 and the fact that $\omega \in A_{s}$, we thus obtain

$$
\begin{aligned}
& \left(\int_{B\left(x_{0}, 2^{j+1} s\right)}\left|b(y)-b_{B\left(x_{0}, s\right)}\right|^{q^{\prime} s^{\prime}} \omega^{1-s^{\prime}}(y) d y\right)^{1 / q^{\prime} s^{\prime}} \\
& \leq C\|b\|_{*}\left(1+\ln \frac{r}{s}\right)\left(\omega^{1-s^{\prime}}\left(B\left(x_{0}, r\right)\right)\right)^{1 / q^{\prime} s^{\prime}} \\
& \leq C\|b\|_{*} r^{n / q^{\prime}}\left(1+\ln \frac{r}{s}\right) \omega\left(B\left(x_{0}, r\right)\right)^{-(1 / p)} .
\end{aligned}
$$

Hence

$$
\begin{aligned}
I_{2} \leq C & \|b\|_{*} \\
& \cdot \int_{2 s}^{\infty}\left(1+\ln \frac{r}{s}\right)\|f\|_{L^{p}\left(\omega, B\left(x_{0}, r\right)\right)} \\
& \cdot \omega\left(B\left(x_{0}, r\right)\right)^{-(1 / p)} \frac{d r}{r} .
\end{aligned}
$$

Therefore

$$
\begin{aligned}
& \left\|I_{2}\right\|_{L^{p}\left(\omega, B\left(x_{0}, s\right)\right)} \\
& \leq C\|b\|_{*} \omega\left(B\left(x_{0}, s\right)\right)^{1 / p} \\
& \cdot \int_{2 s}^{\infty}\left(1+\ln \frac{r}{s}\right)\|f\|_{L^{p}\left(\omega, B\left(x_{0}, r\right)\right)} \\
& \cdot \omega\left(B\left(x_{0}, r\right)\right)^{-(1 / p)} \frac{d r}{r} .
\end{aligned}
$$

Proof of Theorem 4. Since $f \in M_{\varphi_{1}}^{p}\left(w, \mathbb{R}^{n}\right)$ and $\left(\varphi_{1}, \varphi_{2}\right)$ satisfy (15), for $q^{\prime}<p<\infty$, we have

$$
\begin{gathered}
\int_{s}^{\infty}\left(1+\ln \frac{r}{s}\right)\|f\|_{L^{p}\left(\omega, B\left(x_{0}, r\right)\right)} \omega\left(B\left(x_{0}, r\right)\right)^{-(1 / p)} \frac{d r}{r} \\
\leq \int_{s}^{\infty}\left(1+\ln \frac{r}{s}\right) \frac{\|f\|_{L^{p}\left(\omega, B\left(x_{0}, r\right)\right)}}{\operatorname{ess} \inf _{r<t<\infty} \varphi_{1}\left(x_{0}, t\right) \omega\left(B\left(x_{0}, t\right)\right)^{1 / p}} \\
\cdot \frac{\operatorname{ess}_{\inf _{r<t<\infty} \varphi_{1}\left(x_{0}, t\right) \omega\left(B\left(x_{0}, t\right)\right)^{1 / p}} \frac{d r}{r}}{\omega\left(B\left(x_{0}, r\right)\right)^{1 / p}} \\
\leq C\|f\|_{M_{\varphi_{1}}^{p}\left(w, \mathbb{R}^{n}\right)} \int_{s}^{\infty}\left(1+\ln \frac{r}{s}\right) \\
\cdot \frac{\operatorname{ess}_{\inf _{r<t<\infty} \varphi_{1}\left(x_{0}, t\right) \omega\left(B\left(x_{0}, t\right)\right)^{1 / p}} \frac{d r}{r}}{\omega\left(B\left(x_{0}, r\right)\right)^{1 / p}} \\
\leq C\|f\|_{M_{\varphi_{1}}^{p}\left(w, \mathbb{R}^{n}\right)} \varphi_{2}\left(x_{0}, s\right) .
\end{gathered}
$$

Then from (62) we get

$$
\begin{aligned}
\left\|\left[b, \mu_{\Omega}\right](f)\right\|_{M_{\varphi_{2}}^{p}\left(w, \mathbb{R}^{n}\right)} & \\
\leq & C \sup _{x_{0} \in \mathbb{R}^{n}, s>0} \frac{1}{\varphi_{2}\left(x_{0}, s\right)} \\
& \cdot\left(\frac{1}{w\left(B\left(x_{0}, s\right)\right)} \int_{B\left(x_{0}, s\right)}\left|\left[b, \mu_{\Omega}\right](f)(y)\right|^{p} w(y) d y\right)^{1 / p} \\
\leq & C\|b\|_{*} \sup _{x_{0} \in \mathbb{R}^{n}, s>0} \frac{1}{\varphi_{2}\left(x_{0}, s\right)} \\
& \cdot \int_{s}^{\infty}\left(1+\ln \frac{r}{s}\right)\|f\|_{L^{p}\left(\omega, B\left(x_{0}, r\right)\right)} \\
& \cdot \omega\left(B\left(x_{0}, r\right)\right)^{-(1 / p)} \frac{d r}{r} \\
\leq & C\|b\|_{*}\|f\|_{M_{\varphi_{1}}^{p}\left(w, \mathbb{R}^{n}\right)} \cdot
\end{aligned}
$$

\section{Conflict of Interests}

The authors declare that they have no conflict of interests.

\section{Authors' Contribution}

The paper is a joint work of all the authors who contributed equally to the final version of the paper. All authors read and approved the final paper.

\section{Acknowledgments}

The authors are very grateful to the anonymous referees and the editor for their insightful comments and suggestions. The work was supported by the National Natural Science Foundation of China (Grant no. 61340014). 


\section{References}

[1] Y. Ding, D. Fan, and Y. Pan, "Weighted boundedness for a class of rough Marcinkiewicz integrals," Indiana University Mathematics Journal, vol. 48, no. 3, pp. 1037-1055, 1999.

[2] Y. Ding, S. Lu, and K. Yabuta, "On commutators of Marcinkiewicz integrals with rough kernel," Journal of Mathematical Analysis and Applications, vol. 275, no. 1, pp. 60-68, 2002.

[3] C. B. Morrey, "On the solutions of quasi-linear elliptic partial differential equations," Transactions of the American Mathematical Society, vol. 43, no. 1, pp. 126-166, 1938.

[4] D. K. Palagachev and L. G. Softova, "Singular integral operators, Morrey spaces and fine regularity of solutions to PDE's," Potential Analysis, vol. 20, no. 3, pp. 237-263, 2004.

[5] M. A. Ragusa, "Regularity of solutions of divergence form elliptic equations," Proceedings of the American Mathematical Society, vol. 128, no. 2, pp. 533-540, 2000.

[6] G. di Fazio and M. A. Ragusa, "Interior estimates in Morrey spaces for strong solutions to nondivergence form equations with discontinuous coefficients," Journal of Functional Analysis, vol. 112, no. 2, pp. 241-256, 1993.

[7] D. Fan, S. Lu, and D. Yang, "Regularity in Morrey spaces of strong solutions to nondivergence elliptic equations with VMO coefficients," Georgian Mathematical Journal, vol. 5, no. 5, pp. 425-440, 1998.

[8] F. Chiarenza and M. Frasca, "Morrey spaces and HardyLittlewood maximal function," Rendiconti di Matematica e delle sue Applicazioni, vol. 7, no. 3, pp. 273-279, 1987.

[9] T. Mizuhara, "Boundedness of some classical operators on generalized Morrey spaces," in ICM-90 Satellite Conference Proceedings: Harmonic Analysis, S. Igari, Ed., pp. 183-189, Springer, Tokyo, Japan, 1991.

[10] Y. Komori and S. Shirai, "Weighted Morrey spaces and a singular integral operator," Mathematische Nachrichten, vol. 282, no. 2, pp. 219-231, 2009.

[11] S. S. Aliev and V. S. Guliev, "Boundedness of the parametric Marcinkiewicz integral operator and its commutators on generalized Morrey spaces," Georgian Mathematical Journal, vol. 19, no. 2, pp. 195-208, 2012.

[12] V. S. Guliyev, "Generalized weighted Morrey spaces and higher order commutators of sublinear operators," Eurasian Mathematical Journal, vol. 3, no. 3, pp. 33-61, 2012.

[13] R. Wheeden and A. Zygmund, Measure and Integral: An Introduction to Real Analysis, vol. 43 of Pure and Applied Mathematics, Marcel Dekker, New York, NY, USA, 1977.

[14] B. Muckenhoupt, "Weighted norm inequalities for the Hardy maximal function," Transactions of the American Mathematical Society, vol. 165, pp. 207-226, 1972.

[15] J. García-Cuerva and J. L. R. de Francia, Weighted Norm Inequalities and Related Topics, North-Holland Publishing, Amsterdam, The Netherlands, 1985.

[16] F. John and L. Nirenberg, "On functions of bounded mean oscillation," Communications on Pure and Applied Mathematics, vol. 14, pp. 415-426, 1961. 


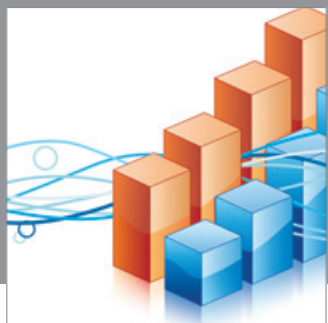

Advances in

Operations Research

mansans

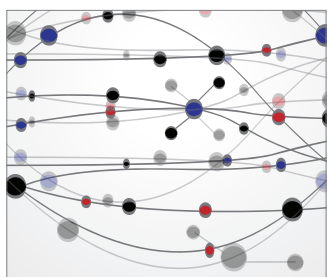

The Scientific World Journal
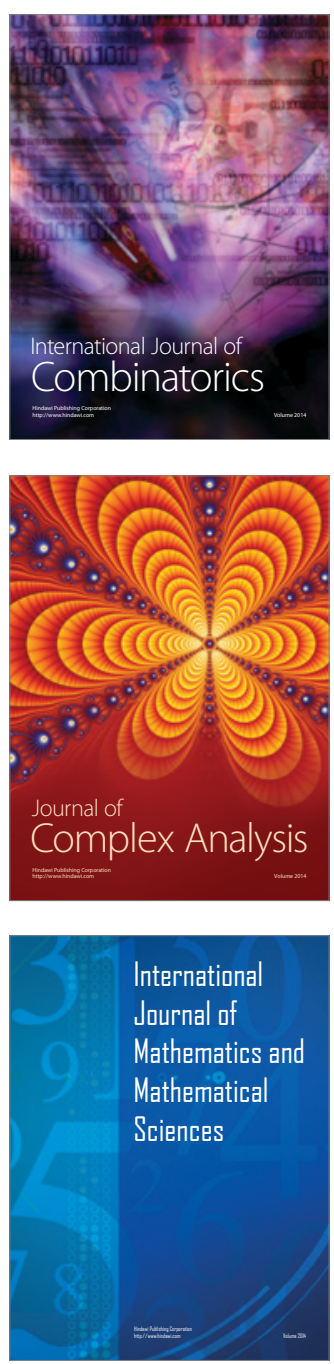
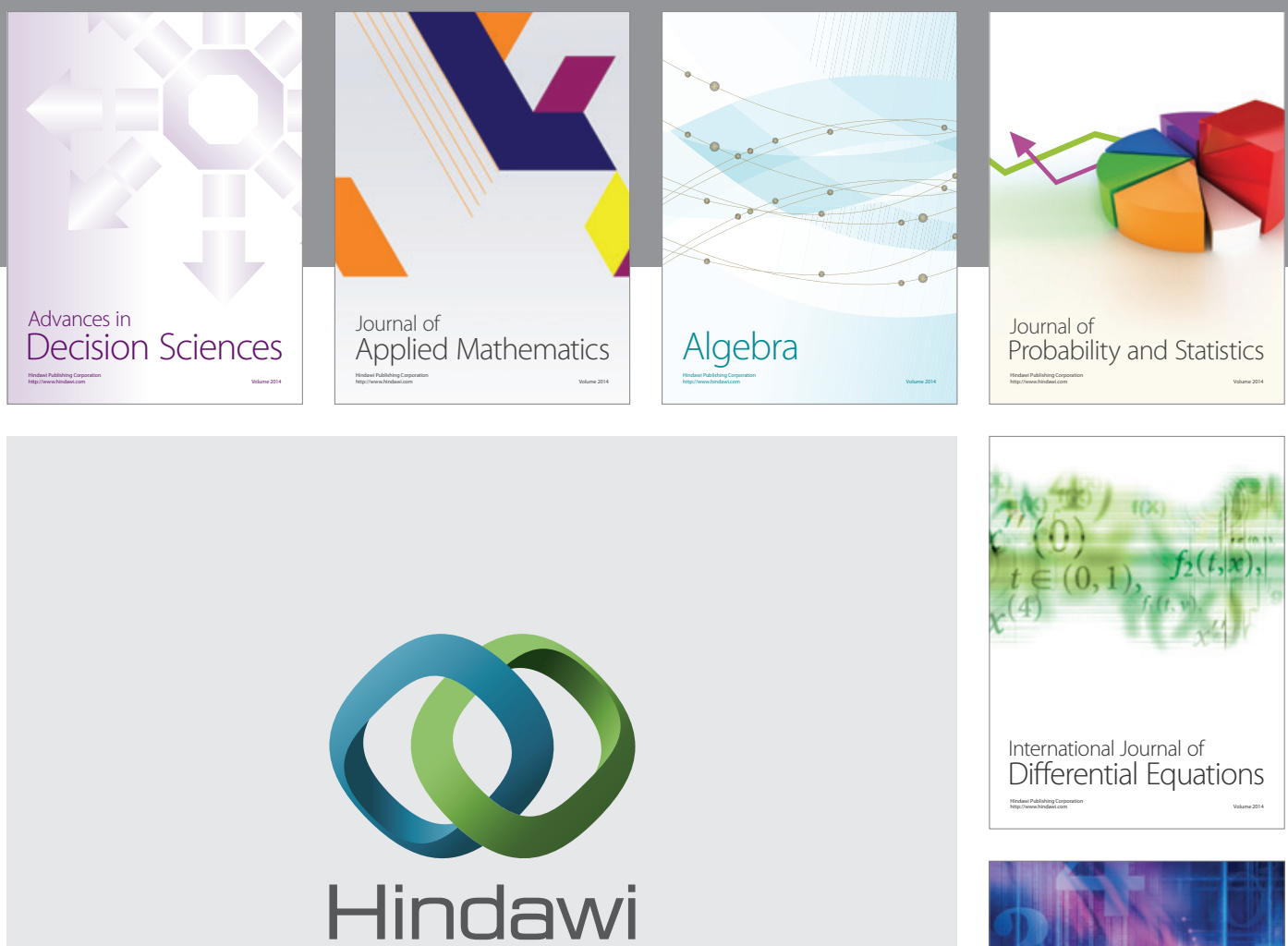

Submit your manuscripts at http://www.hindawi.com
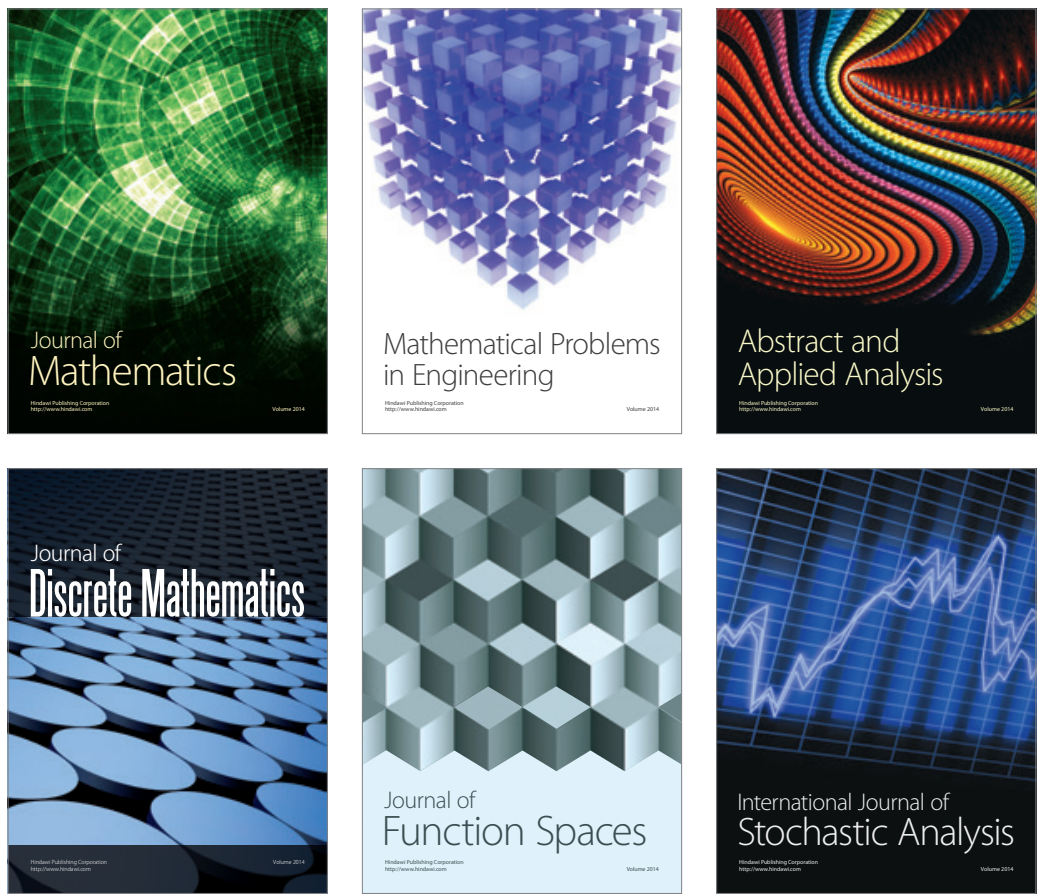

Journal of

Function Spaces

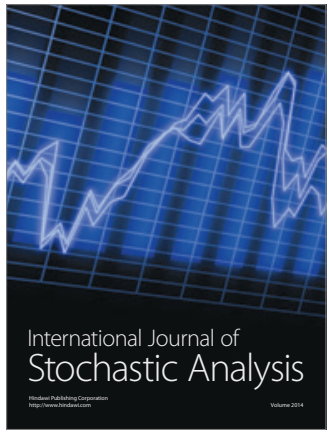

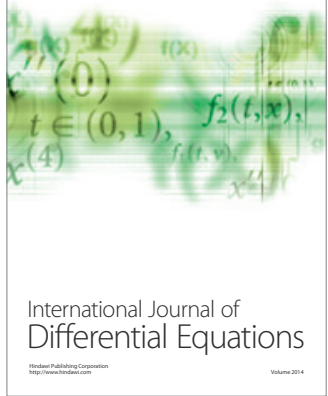
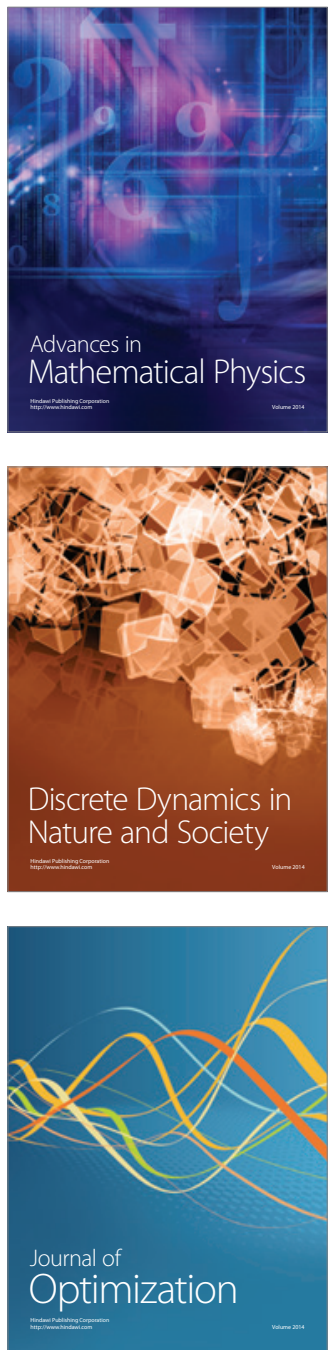